\title{
Four pitfalls in sleep and memory research and how to avoid them
}

Dezso Nemeth $^{1,2,3, *}$, Emilie Gerbier ${ }^{4, *}$, Karolina Janacsek ${ }^{2,3, *}$

${ }^{1}$ Lyon Neuroscience Research Center (CRNL), INSERM U1028, CNRS UMR5292,

Université Lyon 1, Université de Lyon, Lyon, France

${ }^{2}$ Institute of Psychology, ELTE Eotvos Lorand University, Budapest, Hungary

${ }^{3}$ Institute of Cognitive Neuroscience and Psychology, Research Centre for Natural Sciences, Hungarian Academy of Sciences, Budapest, Hungary

${ }^{4}$ Université Côte d'Azur, CNRS, BCL, Nice, France

* These authors contributed equally to this work.

Author Note: Correspondence and requests for material should be addressed to Prof. Dezso Nemeth, CRNL - Lyon Neuroscience Research Center, Université Claude Bernard Lyon 1, Lyon, France. Address: CRNL - Centre Hospitalier Le Vinatier - Bâtiment 462 - 95 Bd Pinel - 69675 Bron Cedex - France. Phone: +33481 106546. 


\begin{abstract}
Understanding the complex relationship between sleep and memory is one of the biggest challenges in neuroscience. Thousands of studies on memory consolidation suggest that sleep triggers offline memory processes, resulting in less forgetting in declarative memory and performance improvement in non-declarative memory. However, an increasing number of contradictory findings reveal potential issues with how research is conducted in this field, that weaken the reliability of these results. Here we describe four methodological pitfalls with respect to experimental designs and statistical analyses that should be avoided in order to unveil the true effect of sleep on memory consolidation: non-optimal experimental designs, task complexity, fatigue effect in repetitive tasks, and data analysis and availability. We then offer solutions that can be used in future research of sleep-dependent consolidation and also more broadly in memory research.
\end{abstract}

Keywords: long-term memory, sleep, consolidation, fatigue effect, circadian effect, napping 


\section{Introduction}

There is a great common interest for sleep in modern societies. The critical influence of sleep on our health and some aspects of our cognition is revealed both by sheer self-observation and by science; moreover, our modern lifestyle and new technologies affect our sleep habits and quality in new ways every day, increasing the prevalence of sleep deprivation and bad sleep hygiene. Memory is also a focus of societal interest, with respect to education and learning on one side of the developmental spectrum, and to aging and Alzheimer's disease on the other. Following this great interest in sleep as well as in memory, the effect of sleep on memory has gained an increasing amount of attention in psychology and neuroscience research in the last two decades, with thousands of dedicated publications. Additionally, a number of theories and models explaining the effect of sleep on memory have also been developed (Ackermann \& Rasch, 2014; Antony, Schönauer, Staresina, \& Cairney, 2019; Boyce, Williams, \& Adamantidis, 2017; Diekelmann \& Born, 2010; Diekelmann, Wilhelm, \& Born, 2009; Feld \& Born, 2017; Lewis \& Durrant, 2011; Mednick, Cai, Shuman, Anagnostaras, \& Wixted, 2011; Saletin \& Walker, 2012; Siegel, 2001; Stickgold \& Walker, 2005, 2013; Tononi \& Cirelli, 2006; Tononi \& Cirelli, 2014; Walker, 2005). In this article the focus will lie on the effect of sleep on memory consolidation. That is, how sleeping after having learned something - e.g., new vocabulary or playing the piano - increases subsequent memory, compared to an equivalent time spent without sleep.

According to the often-cited empirical studies (e.g., Gais, Lucas, \& Born, 2006; Walker, Brakefield, Hobson, \& Stickgold, 2003) and reviews on this topic (Diekelmann et al., 2009; King, Hoedlmoser, Hirschauer, Dolfen, \& Albouy, 2017; Rasch \& Born, 2013), in healthy adults declarative memories are less forgotten after an episode of sleep than after a similar episode of wakefulness, whereas non-declarative memories are improved after sleep. The evidence for sleep-related memory consolidation is so convincing that it has been claimed that 
"While memory formation is not the only function of sleep, it seems to be the most important (...)" (Born \& Wilhelm, 2012, p. 192) or that "(...) active system consolidation might be an evolutionary conserved function of sleep." (Vorster \& Born, 2015, p. 103). Here we claim that such statements, particularly for non-declarative memory, are not supported by solid evidence, at least partly due to experimental and methodological issues. Indeed, there have been discussions as to the actual impact of sleep on memory consolidation (e.g., Mantua, 2018; Pan \& Rickard, 2015; Vertes \& Siegel, 2005). A non-negligible number of studies did not actually find sleep-related consolidation effects, especially for non-declarative memory (e.g., Csabi, Varszegi-Schulz, Janacsek, Malecek, \& Nemeth, 2014; Nemeth et al., 2010; Robertson, Pascual-Leone, \& Press, 2004; Song, Howard, \& Howard, 2007; Viczko, Sergeeva, Ray, Owen, \& Fogel, 2018; Wilson, Baran, Pace-Schott, Ivry, \& Spencer, 2012). In addition, the correlations observed between sleep physiology and memory consolidation are often not replicated across studies and are sometimes too numerous to reliably interpret the significant ones. Moreover, they sometimes go in the opposite direction as to what is expected (Mantua, 2018).

Given this controversial state of the art, we propose here a synthetic methodological guideline for future research on sleep-related consolidation. Such a comprehensive guideline is lacking so far, hindering the progress in our understanding of the effect of sleep on memory in fields ranging from psychology to biology and neuroscience. We highlight four critical pitfalls that could be responsible for the contradictory findings in the literature, and then propose solutions to prevent them and guide future research.

\section{Pitfall 1: Non-optimal experimental designs}

In this section we identify four areas that could benefit from improvements in the experimental designs and suggest solutions to each of them. Figure 1 illustrates this issue and its solutions. 


\section{1a) Studies with pathological populations}

Studies investigating the effect of sleep disorders (e.g., primary insomnia, obstructive sleep apnea, sleep-disordered breathing) on sleep-related consolidation typically compare a pathological group with a control healthy group and often use only an PM-AM design (for reviews, see Ahuja et al., 2018; Cellini, 2017). In such designs, learning takes place in the evening and performance is tested in the following morning (e.g., Backhaus et al., 2006; Csabi et al., 2014). Importantly, however, if patients show a weaker performance than the control group (that is, less off-line improvement in non-declarative memory or more forgetting in declarative memory), one cannot disentangle whether it is caused by the actual overnight sleep (i.e., state-dependent consolidation) or by a trait-dependent effect of the sleep disturbances on memory processes involving consolidation but perhaps also encoding and retrieval. (Ahuja et al., 2018; Csábi, Benedek, Janacsek, Katona, \& Nemeth, 2013; Rosenzweig et al., 2015; Wallace \& Bucks, 2013).

\section{Solutions}

Studies comparing sleep-disordered populations and healthy controls should also include an AM-PM condition, that is, learning in the morning and testing in the evening. To demonstrate that the pathology specifically affects sleep-related consolidation, we need to observe not only that test performance or improvement in the PM-AM (sleep) condition is lower in the pathological group than in the control group, but also that test performance or improvement in the AM-PM (wake) condition is similar in both groups. If the overall results are the same in the sleep-disordered and in the control groups, then one can conclude that the sleep pathology does not affect sleep-related consolidation. If test performance or improvement of the pathological group is affected both in the PM-AM and in the AM-PM conditions, then one can rather conclude that the pathology affects more general consolidation processes, not 
specifically related to sleep. Such design has been conducted only in a few studies. For instance, Nissen et al. (2011) compared the effect of PM-AM vs. AM-PM conditions on consolidation of procedural and declarative memory in insomniac and healthy participants. They observed similar consolidation over the wake interval in both groups. However, whereas in the control group consolidation was higher in the sleep than in the wake condition, in the insomniac group it was comparable in the wake and in the sleep conditions. This indicates that insomnia in this group prevented sleep-related consolidation in procedural and declarative memory.

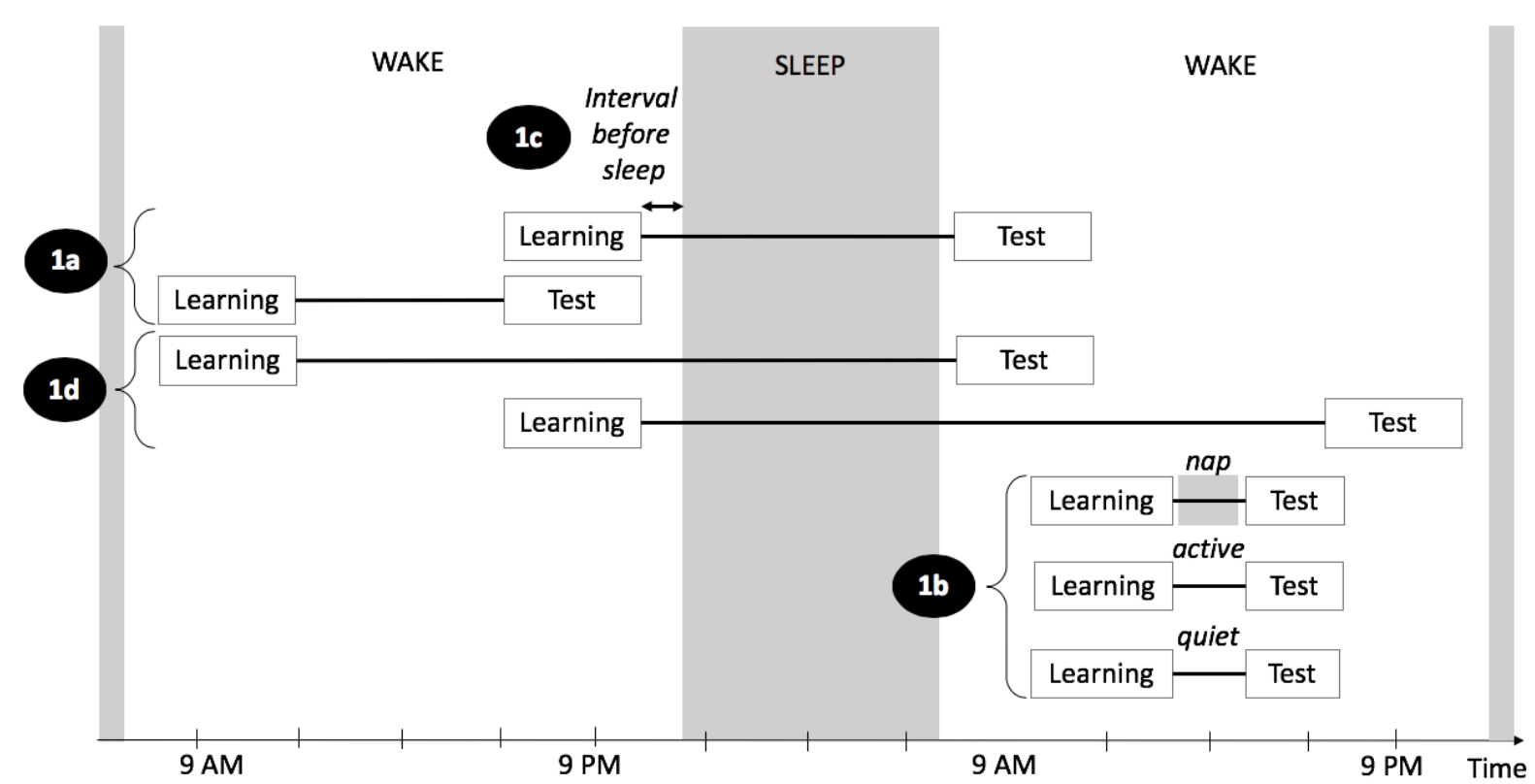

Figure 1: Illustration of the four areas of Pitfall 1 (non-optimal experimental designs). 1a refers to studies with pathological populations, $1 \mathrm{~b}$ refers to appropriate control conditions in napping studies, $1 \mathrm{c}$ refers to time interval between the end of the task and bedtime, and 1d refers to time-of-day effects.

\section{1b) Appropriate control conditions in napping studies}

Napping studies typically compare changes in performance following an interval that includes daytime sleep with an interval that includes awake activities. Such design however does not rule out the possibility that other factors than sleep per se affect memory consolidation. 
For example, reducing external interferences during the post-learning interval may be sufficient to produce off-line consolidation (e.g., Mednick et al., 2011; Wamsley, 2019).

\section{Solutions}

Adding a carefully controlled quiet wake condition (e.g., under dim light while keeping eyes open to avoid falling asleep) helps to distinguish whether sleep is a specific state that triggers off-line improvement (i.e., Sleep > Quiet Rest) or a non-specific state that only protects memories from interferences (i.e., Sleep = Quiet Rest). The benefits of such design has been just recently recognized. Studies using such design led to mixed findings, with some studies showing more consolidation in the nap condition than in the quiet wake condition (Piosczyk et al., 2013; Schichl, Ziberi, Lahl, \& Pietrowsky, 2011; Schönauer, Geisler, \& Gais, 2014) and others showing that quiet rest produced effects on memory consolidation similar to those observed in sleep conditions (Mednick, Makovski, Cai, \& Jiang, 2009; Simor, Zavecz, et al., 2019), suggesting that sleep per se may not be necessary for consolidation but rather only provides a favorable environment. Recent observations that memory reactivations and replay occur not only during sleep but also during quiet rest (Craig \& Dewar, 2018; Schapiro, McDevitt, Rogers, Mednick, \& Norman, 2018; Wamsley, 2019) further highlight the need for such control conditions. (Note that a quiet rest condition would theoretically also be informative in a PM-AM design as discussed in 1a, although it might be extremely difficult to avoid falling asleep in a quiet environment during the night. Therefore, sleep deprivation studies typically use an active wake condition during the night.)

\section{1c) Time interval between the end of the task and bedtime}

The duration of the interval between the task and sleep onset may vary across experiments, conditions and individuals, potentially hindering the assessment of the true effect of sleep. For example, it has been shown that the more time elapses between the end of the 
learning task and sleep onset, the smaller the consolidation effects (Press, Casement, PascualLeone, \& Robertson, 2005; Robertson, Press, \& Pascual-Leone, 2005; Walker et al., 2003). A longer interval may be associated with more retroactive interference if the subject engages in activities that interfere with the learned information by recruiting the same cognitive processes or neural network.

\section{Solutions}

Attention should be paid to this issue when designing experiments. Experiments should control the duration of the interval between the experimental task and bedtime, as well as the participants' activities during this interval. Additionally, if experiments aim to minimize retroactive interference (and thus to observe "pure" effects of sleep), participants should go to bed as soon as possible after the learning tasks. Such designs are more feasible when participants sleep in the lab during the experiment. If, however, participants sleep at home after the learning session, at least post-experiment questionnaires or mobile actigraphy should be employed to assess the duration of this interval and the activities performed, and this information should be appropriately taken into account in data analysis.

\section{1d) Time-of-day effects}

It has been shown that learning and memory performance is affected by the time of day when the memory task is performed (Schmidt, Collette, Cajochen, \& Peigneux, 2007). Timeof-day effects can lead to potential confounds in sleep-related consolidation studies. For example, a greater off-line improvement in a PM-AM condition compared to an AM-PM condition may be, at least partially, caused by weaker performance in the evening (when learning takes place in the PM-AM condition) due to circadian effects or fatigue (Keisler, Ashe, \& Willingham, 2007), and a better performance after a night's sleep in the morning when participants are well rested. In this case, it is unclear how much sleep per se contributes to improved performance compared to these circadian effects. 


\section{Solutions}

These potential time-of-day or circadian effects are not easy to control because in humans sleep typically occurs during the night (although see Tucker et al. (2017) for an inverted 12-hour schedule with sleep occurring during daytime). A possible solution is to focus on the effect of napping on learning and memory performance, as in this case training and testing occur at the same time in the nap and awake groups (see 2b). Note, however, that daytime sleep and night sleep can affect memory differently (Payne et al., 2015).

When focusing on the effects of nighttime sleep on memory consolidation, e.g., in a PM-AM vs. AM-PM design, additional control groups should be tested to disentangle the circadian effects from the effect of sleep per se. Thus, a full design would include groups in AM-PM (12h Wake), PM-AM (12h Sleep), PM-PM (24h Sleep early) and AM-AM (24h Sleep late) conditions (e.g., Fenn, Nusbaum, \& Margoliash, 2003; Hallgato, Győri-Dani, Pekár, Janacsek, \& Nemeth, 2013; Talamini, Nieuwenhuis, Takashima, \& Jensen, 2008; Tucker, McKinley, \& Stickgold, 2011). A difference in learning performance when learning takes place during the evening (in PM-AM and PM-PM conditions) vs. in the morning (in AM-PM and AM-AM conditions) would indicate potential circadian effects and preclude further interpretation about the effect of sleep on consolidation. The pattern of results required to demonstrate a beneficial effect of sleep without any time-of-day effect is an absence of difference during the learning session in the four conditions, and the following pattern of performance during the testing session: PM-AM > AM-PM and PM-PM = AM-AM. It should be noted, however, that sleep shortly after learning may be more beneficial to consolidation than sleep that occurs later after learning during the 24-h interval (e.g., Gais et al., 2006; Talamini et al., 2008), which would result in PM-PM > AM-AM ) (Figure 1d). 


\section{Pitfall 2: Task complexity}

A great difficulty in sleep and memory research, and in cognitive neuroscience and psychology in general, is that practically every task involves several cognitive processes (e.g., Jacoby, 1991; Sigman \& Dehaene, 2005). The learning/memory scores that are used to assess behavioral performances typically reflect a mixture of these cognitive processes (Cohen, Pascual-Leone, Press, \& Robertson, 2005). For example, even a simple perceptual-motor learning task may require processing of perceptual stimuli, their serial order and/or their transitional probabilities, perceptual-motor coordination, selective attention, and other processes/functions. However, different types of consolidation processes may be involved depending on which cognitive processes are involved in a given task, and to what extent (e.g., Conte \& Ficca, 2013; King et al., 2017; Stickgold, 2013; Stickgold \& Walker, 2013). It is highly plausible that sleep affects the distinct cognitive processes differently, and this is sometimes neglected in current sleep and memory research. Moreover, other external factors such as subject characteristics may also influence sleep-dependent consolidation.

\section{Solutions}

Since there are no process-pure learning/memory tasks, attention should be paid to the specific cognitive processes that are involved in a particular task. We recommend that future research aiming at understanding the specific effect of sleep on consolidation should disentangle these different cognitive processes using experimental designs and data-analyses, and examine whether they are differentially affected by sleep. For example, within procedural learning tasks, allocentric vs. egocentric representations (Viczko et al., 2018), perceptual vs. motor components of learning (Hallgato et al., 2013), acquisition of statistical vs. sequential regularities (Simor, Zavecz, et al., 2019) can be disentangled, and may be differently affected by sleep (see also Albouy et al., 2013; Cohen et al., 2005; Song \& Cohen, 2014). In addition, a 
great variety of variables, including individual characteristics, motivation, and strategies, should also be assessed and controlled for (Conte \& Ficca, 2013).

\section{Pitfall 3: Fatigue effect in repetitive tasks}

Some studies, specifically those investigating non-declarative procedural learning, use tasks that involve continuous practice with a series of repetitions of the same action, for example, pressing keys (M. J. Nissen \& Bullemer, 1987). Learning is measured as the improvement in accuracy or in reaction time to perform the task. Usually, the performance at the end of the training session serves as a baseline to measure improvement at the test session that takes place after an interval occupied by sleep or wakefulness. Yet, after a certain amount of time spent performing the task, the subject's observed improvement is less marked, which can be interpreted as a reactive inhibition effect that reflects the built-up of fatigue over the trials (e.g., Brawn, Fenn, Nusbaum, \& Margoliash, 2010; Pan \& Rickard, 2015). This effect often results in smaller improvement or even an increase in reaction times as the task progresses. This effect then disappears after a certain period of time spent without performing the task. Thus, the actual performance after extended practice is not representative of genuine expertise in the task and, therefore, comparing the performance at the test session with that of the end of the training session may lead to illusory sleep-related effect. Figure 2 (upper panel) illustrates this issue. In several cases, after eliminating this reactive inhibition effect by releasing the presumed fatigue, the sleep-related off-line improvement was no longer observed (e.g., Cai \& Rickard, 2009; Rickard, Cai, Rieth, Jones, \& Ard, 2008). Although this issue is primarily relevant in procedural learning studies, it is possible that reactive inhibition also affects performance in declarative memory studies, particularly if they include repetitive presentations of the same items or a long period of memorization. 


\section{Solutions}

To address this issue, we highlight solutions that concern both experimental designs and data analyses.

\section{Experimental design solutions}

a) Using a post-rest performance at the end of the training session as a baseline. Resting for a few minutes after the training session appears to be sufficient to wash out the effect of reactive inhibition on performance. Measures of performance after a break is therefore a more appropriate baseline to assess subsequent off-line consolidation (e.g., Brawn et al., 2010; Simor, Zavecz, et al., 2019). Figure 2 (middle panel) illustrates this solution.

b) Learning through spaced practice rather than massed practice. Introducing regular short rest intervals during the training session (often termed spaced practice) impedes the accumulation of reactive inhibition compared to experimental designs that use massed practice in which such rest intervals are not included (e.g., Brawn et al., 2010; Rickard et al., 2008; Rieth, Cai, McDevitt, \& Mednick, 2010). Figure 2 (lower panel) illustrates this solution. 

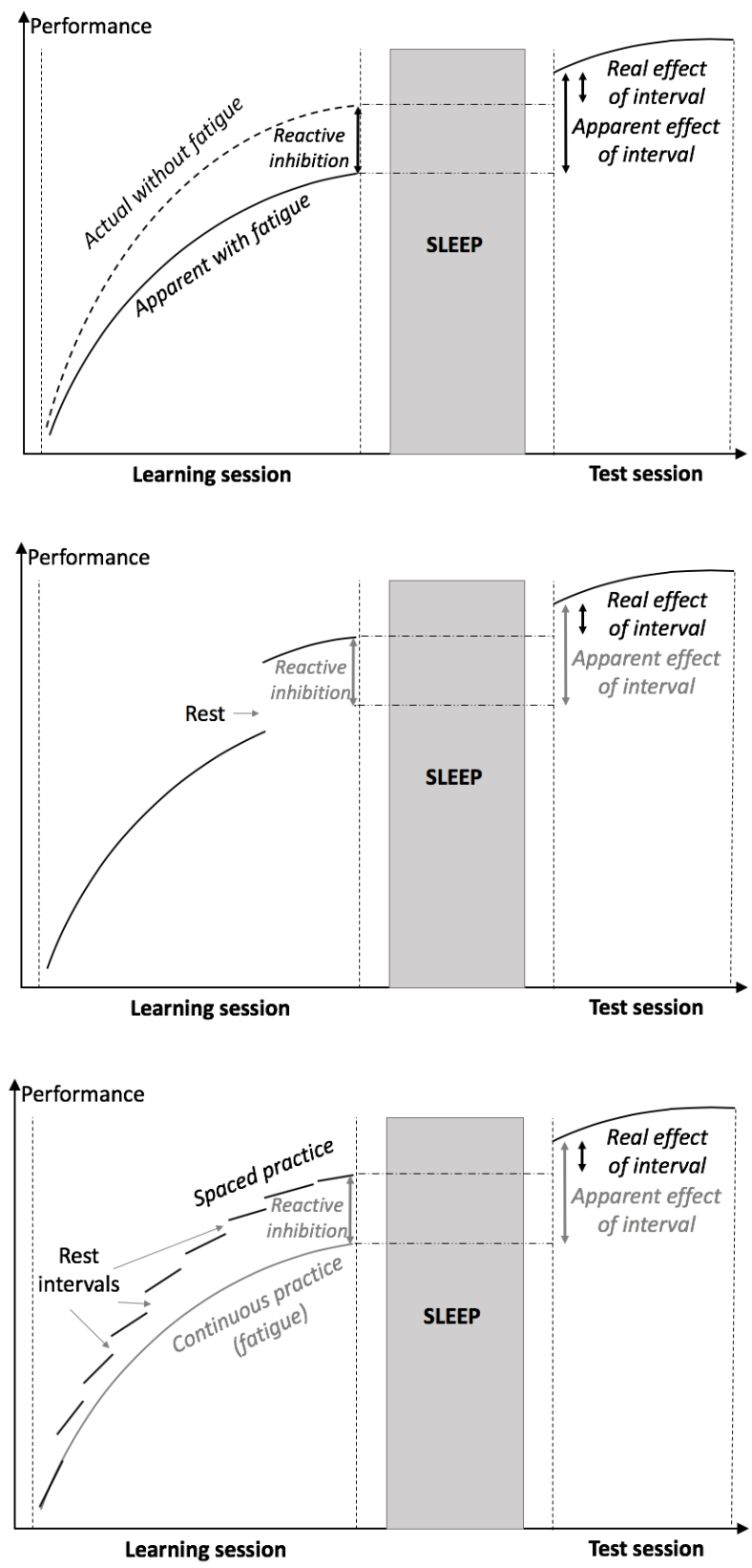

Figure 2: The fatigue effect (upper panel), the short rest solution (middle) and the spaced-practice solution (lower)

\section{Data analysis solutions}

a) Using curve fitting methods. Here we highlight two such methods. First, a functionbased model (e.g., a power function for reaction time improvement) can be elaborated on the training session and used to predict future performance, enabling the comparison between the predicted and the actual outcomes measured during the test session. This way, one avoids averaging over data points which may mask the real final performance, if there is still an 
improvement, at the end of the training phase (Pan \& Rickard, 2015). Second, a function can be fitted to the training session data and then a continuity test can be used in the test session to evaluate whether the performance is a simple continuation from the training session or whether there is an abrupt change between the sessions (see details on these approaches in Pan \& Rickard, 2015).

b) Performing computational modeling. Using computational models on trial-by-trial data can help overcome the issue of fatigue by directly including reactive inhibition as a separate parameter in the model. For instance, in a probabilistic sequence learning task, Török et al. (2017) used such model allowing the estimation of the actual magnitude of learning, independent of the effect of reactive inhibition. Such models can be used in a wide range of learning and memory tasks, including finger tapping and other sequence learning tasks.

c) Excluding data from the second part of each task block. Because of fatigue, performance declines between the first and the second half of a given task block. Using only the data from the first part of the blocks would reflect a more accurate estimation of genuine expertise in the task (Nemeth et al., 2013; Rickard et al., 2008).

\section{Pitfall 4: Data analyses and availability}

The sleep-related consolidation studies could benefit from an update in data analysis practices; which are currently evolving in the scientific community in general.

\section{4a) Determining sample size and reporting non-significant results}

In the field of sleep-related consolidation, studies typically use samples with around 20 participants per group, often between 12 and 20 (e.g., Gais et al., 2006; Wagner, Hallschmid, Rasch, \& Born, 2006) and in some studies even less (e.g., Csabi et al., 2015), which may be due to complicated or demanding study designs, difficulties recruiting clinical populations, or drop-out of participants (i.e., experimental attrition). Importantly, sample sizes are usually not 
being determined by a priori power analyses that are based on the expected effect sizes. The resulting small sample sizes may involve a lack of power when using inferential statistics, potentially leading to Type 2 errors, that is, not detecting an effect that exists. On the other hand, a common but inappropriate practice when additional data is collected until a significant effect is reached (p-hacking) potentially leads to Type 1 errors, that is, the detection of an effect that does not exist. In addition, if one wants to conclude that sleep has no effect on some aspects of memory consolidation, such conclusion cannot be drawn by showing non-significant results in classical statistical analysis approaches (t-test, ANOVA, correlation, etc.). Of note, such nonsignificant effects are often observed when consolidation results in stabilization of the acquired knowledge without forgetting or performance improvement (i.e., no performance change). Such consolidation effects can be present independently of sleep and, thus, the present pitfall may be relevant for a wider range of neuroscience and psychology studies, outside those focusing on sleep per se.

\section{Solutions}

a) Before data collection. It has long been recommended in guidelines (e.g., American Psychological Association) that experimenters should determine the sample size before starting the experiment by computing power analyses based on the magnitude of the effect size estimated or found in previous studies that observed similar effects.

b) During data collection. For particularly costly experimental protocols, Bayesian analyses (Dienes, 2016; Dienes, Coulton, \& Heather, 2018; Dienes \& Mclatchie, 2018; Wagenmakers et al., 2018) computed in the course of data collection can be used to know whether there is enough evidence in favor of a given a priori defined effect so that one can stop data collection (Rouder, 2014; Schönbrodt, Wagenmakers, Zehetleitner, \& Perugini, 2017).

c) After data collection. Bayesian analyses, in particular the Bayes Factors, are rarely reported in the field of memory consolidation (see Brown \& Maylor, 2017for an exception) 
whereas they are increasingly reported in other areas of psychology and neuroscience. The Bayes Factor indicates an odd ratio of relative probabilities in favor of the null hypothesis (i.e., the absence of a difference between the conditions or group means) vs. in favor of the alternative hypothesis (i.e., the difference between the conditions or group means) (Jarosz \& Wiley, 2014; Rouder, Speckman, Sun, Morey, \& Iverson, 2009). Bayesian statistics allow a more finegrained quantitative evaluation of the effect of sleep on consolidation and, contrary to p-value based inferential statistics, enable the demonstration of the absence of an effect (Bogacz, Wagenmakers, Forstmann, \& Nieuwenhuis, 2009; Dienes, 2014). This is useful in the sleep and memory literature in cases where there is no sleep effect or no change in performance (neither forgetting nor improvement).

\section{4b) Correlations between sleep parameters and memory consolidation}

Beyond the comparison of groups or conditions, conclusions for the effect of sleep are often based on correlations between behavioral performances and sleep polysomnographic parameters (e.g., Scullin, 2013; Simor, Zavecz, et al., 2019). However, it has been raised that some of these correlation analyses are a consequence of suboptimal statistical practices, leading to spurious correlations (e.g., Mantua, 2018; Pan \& Rickard, 2015). Small sample sizes can also lead to such spurious correlations. When sufficiently large sample sizes are used, they may disappear. For example, Ackermann et al. (2015) did not find any significant correlation between sleep and memory performance in a sample of 929 participants.

\section{Solutions}

The correlations to be computed should be planned a priori and corrected for multiple comparisons in order to avoid increases in Type 1 errors (Abdi, 2007). Non-significant planned correlations should also be systematically reported (Forstmeier, Wagenmakers, \& Parker, 2017). If no relationship is expected between certain sleep parameters and behavioral 
performance, Bayesian approaches should be used to draw conclusions in favor of the null hypothesis instead of reporting non-significant results (see 4a).

\section{4c) Access to and re-analysis of previous data}

Other fields of neuroscience using techniques such as neuroimaging (fMRI) have engaged in open science initiatives by, for example, depositing raw data in open-access databases (e.g., OpenfMRI; Poldrack \& Gorgolewski, 2017), which has recently been extended to other neuroimaging and electrophysiology methods such as EEG as well (OpenNEURO; Gorgolewski, Esteban, Schaefer, Wandell, \& Poldrack, 2017). However, sleep and memory research is lagging behind with respect to this practice. Making data publicly available enables the re-analysis of old data when new analysis techniques and/or new theories are developed. For example, it has only recently been recognized that the rapid-eye-movement (REM) stage of sleep is not a unified state; instead there are at least two different types of REM, each of them with different characteristics (Simor, Gombos, Blaskovich, \& Bódizs, 2017; Simor, van Der Wijk, Gombos, \& Kovács, 2019). Access to previous sleep EEG data would make it possible to specifically test the role of these two REM types in memory consolidation. Additionally, these open databases can also support reliable synthesis of the data through meta-analyses with larger sample sizes. Thus, overall, publicly available sleep EEG and behavioral data could resolve previous issues of spurious results and can further advance the field.

\section{Solutions}

To maximize the benefits of previous research in the scientific community, we suggest that researchers engage in open science (Nosek et al., 2015) and make data publicly available. There are several online open repositories available for such purposes, such as Open Science Framework or OpenNeuro.org. In particular, developing a specific open database for sleep 
research with EEG, polysomnographic and behavioral data would greatly benefit sleep and memory research.

\section{Conclusion}

We highlighted four critical methodological pitfalls that impede research in the field of sleep and memory and we offered solutions to prevent them. They are presented in Table 1 . We believe that following the recommendations of the present guideline can contribute to more reliable results in future studies of this field so that the genuine effect of sleep on memory can be established. The present article can also help bridge gaps between disciplines (e.g., cognitive psychology, neuropsychology, biology, neuroscience) that have different research traditions in order to make outstanding progress in our understanding of the links between memory and sleep and to provide answers to the society's interest on this topic. We also suggest that some of the pitfalls described here (i.e., the fatigue effect, task complexity, and statistical and data availability issues) are relevant not only in sleep research but also in memory research more broadly. 
Table 1: Summary of the pitfalls and their solutions

\begin{tabular}{|c|c|}
\hline Pitfall & Solutions \\
\hline $\begin{array}{l}\text { Non-optimal experimental } \\
\text { designs } \\
\text { May lead to inaccurate } \\
\text { conclusions due to } \\
\text { confounding variables }\end{array}$ & $\begin{array}{l}\text { a) Studies with pathological populations: including an AM-PM } \\
\text { condition (control for individual consolidation capacity) } \\
\text { b) Napping studies: including a quiet-rest control group (control for } \\
\text { interference) } \\
\text { c) Time interval between end of task and bedtime (all studies): } \\
\text { Controlling for duration of and subject's activity during the interval } \\
\text { between end of task and sleep (control for interferences) } \\
\text { d) Nighttime sleep studies: using a full 4-condition design (AM-PM; } \\
\text { PM-AM; AM-AM; PM-PM) with 24-h intervals (control for time-of } \\
\text { day effects) }\end{array}$ \\
\hline $\begin{array}{l}\text { Task complexity } \\
\text { Every task involves multiple } \\
\text { cognitive processes }\end{array}$ & $\begin{array}{l}\text { Disentangling different cognitive processes of interest by experimental } \\
\text { designs and data-analyses, and examine whether they are differentially } \\
\text { affected by sleep and consolidation. }\end{array}$ \\
\hline $\begin{array}{l}\text { Fatigue effect in repetitive } \\
\text { tasks } \\
\text { May lead to spurious } \\
\text { boosting effects of sleep by } \\
\text { negatively affecting } \\
\text { performance after continuous } \\
\text { practice }\end{array}$ & $\begin{array}{l}\text { Experimental design: } \\
\text { a) Using a post-rest performance at the end of the training session } \\
\text { as a baseline } \\
\text { b) Learning through spaced practice rather than massed practice } \\
\text { Data analysis: } \\
\text { a) Using curve fitting methods } \\
\text { b) Performing computational modeling } \\
\text { c) Excluding data from the second part of the task blocks }\end{array}$ \\
\hline $\begin{array}{l}\text { Data analyses and } \\
\text { availability } \\
\text { Inappropriate analyses may } \\
\text { lead to spurious conclusions; } \\
\text { non-available data impedes } \\
\text { research progress }\end{array}$ & $\begin{array}{l}\text { a) Determining required sample size and reporting non- } \\
\text { significant results. Determine the required sample size a priori and/or } \\
\text { use Bayesian analyses to decide when to stop data collection (avoid p- } \\
\text { hacking). Report Bayes Factors (to quantify the conclusion and argue for } \\
\text { the null hypothesis) } \\
\text { b) Correlations between sleep parameters and memory } \\
\text { consolidation. Plan correlation analyses of interest in advance, correct } \\
\text { for multiple comparisons, and report non-significant planned } \\
\text { comparisons } \\
\text { c) Access to and re-analysis of previous data. Make data } \\
\text { available through open science platforms (enabling re-analyses of } \\
\text { previous data) }\end{array}$ \\
\hline
\end{tabular}




\section{Acknowledgements}

This research was supported by the National Brain Research Program (project 2017-1.2.1-NKP2017-00002); Hungarian Scientific Research Fund (NKFIH-OTKA K 128016, PI: D. N., NKFIH-OTKA PD 124148, PI: K.J.); Janos Bolyai Research Fellowship of the Hungarian Academy of Sciences (to K. J.); IDEXLYON Fellowship (to D.N). We are grateful to Kate Schipper and Teodora Vekony for her help and comments on the manuscript.

\section{References}

Abdi, H. (2007). Bonferroni and Šidák corrections for multiple comparisons. Encyclopedia of measurement and statistics, 3, 103-107.

Ackermann, S., Hartmann, F., Papassotiropoulos, A., de Quervain, D. J., \& Rasch, B. (2015). No associations between interindividual differences in sleep parameters and episodic memory consolidation. Sleep, 38(6), 951-959.

Ackermann, S., \& Rasch, B. (2014). Differential effects of non-REM and REM sleep on memory consolidation? Current Neurology and Neuroscience Reports, 14(2), 430.

Ahuja, S., Chen, R. K., Kam, K., Pettibone, W. D., Osorio, R. S., \& Varga, A. W. (2018). Role of normal sleep and sleep apnea in human memory processing. Nature and science of sleep, 10, 255.

Albouy, G., Fogel, S., Pottiez, H., Nguyen, V. A., Ray, L., Lungu, O., . . Doyon, J. (2013). Daytime sleep enhances consolidation of the spatial but not motoric representation of motor sequence memory. PloS One, 8(1), e52805.

Antony, J. W., Schönauer, M., Staresina, B. P., \& Cairney, S. A. (2019). Sleep spindles and memory reprocessing. Trends in Neurosciences, 42(1), 1-3. 
Backhaus, J., Junghanns, K., Born, J., Hohaus, K., Faasch, F., \& Hohagen, F. (2006). Impaired declarative memory consolidation during sleep in patients with primary insomnia: influence of sleep architecture and nocturnal cortisol release. Biological Psychiatry, 60(12), 1324-1330.

Bogacz, R., Wagenmakers, E.-J., Forstmann, B. U., \& Nieuwenhuis, S. (2009). The neural basis of the speed-accuracy tradeoff. Trends in Neurosciences, 33(1), 10-16.

Born, J., \& Wilhelm, I. (2012). System consolidation of memory during sleep. Psychological Research, 76(2), 192-203.

Boyce, R., Williams, S., \& Adamantidis, A. (2017). REM sleep and memory. Current Opinion in Neurobiology, 44, 167-177.

Brawn, T. P., Fenn, K. M., Nusbaum, H. C., \& Margoliash, D. (2010). Consolidating the effects of waking and sleep on motor-sequence learning. The Journal of neuroscience, 30(42), 13977-13982.

Brown, H., \& Maylor, E. A. (2017). Memory consolidation effects on memory stabilization and item integration in older adults. Psychonomic bulletin \& review, 24(4), 10321039.

Cai, D. J., \& Rickard, T. C. (2009). Reconsidering the role of sleep for motor memory. Behavioral Neuroscience, 123(6), 1153.

Cellini, N. (2017). Memory consolidation in sleep disorders. Sleep Medicine Reviews, 35, $101-112$.

Cohen, D. A., Pascual-Leone, A., Press, D. Z., \& Robertson, E. M. (2005). Off-line learning of motor skill memory: A double dissociation of goal and movement. PNAS, 102(50), $18237-18241$.

Conte, F., \& Ficca, G. (2013). Caveats on psychological models of sleep and memory: a compass in an overgrown scenario. Sleep Medicine Reviews, 17(2), 105-121. 
Craig, M., \& Dewar, M. (2018). Rest-related consolidation protects the fine detail of new memories. Scientific Reports, 8(1), 6857.

Csábi, E., Benedek, P., Janacsek, K., Katona, G., \& Nemeth, D. (2013). Sleep disorder in childhood impairs declarative but not nondeclarative forms of learning. Journal of Clinical and Experimental Neuropsychology, 35(7), 677-685.

Csabi, E., Benedek, P., Janacsek, K., Zavecz, Z., Katona, G., \& Nemeth, D. (2015). Declarative and Non-declarative Memory Consolidation in Children with Sleep Disorder. Frontiers in Human Neuroscience, 9, 709. doi:10.3389/fnhum.2015.00709

Csabi, E., Varszegi-Schulz, M., Janacsek, K., Malecek, N., \& Nemeth, D. (2014). The consolidation of implicit sequence memory in obstructive sleep apnea. PloS One, 9(10), e109010.

Diekelmann, S., \& Born, J. (2010). The memory function of sleep. Nature Reviews Neuroscience, 11(2), 114-126.

Diekelmann, S., Wilhelm, I., \& Born, J. (2009). The whats and whens of sleep-dependent memory consolidation. Sleep Medicine Reviews, 13(5), 309-321.

Dienes, Z. (2014). Using Bayes to get the most out of non-significant results. Frontiers in Psychology, 5. doi:10.3389/fpsyg.2014.00781

Dienes, Z. (2016). How Bayes factors change scientific practice. Journal of Mathematical Psychology, 72, 78-89.

Dienes, Z., Coulton, S., \& Heather, N. (2018). Using Bayes factors to evaluate evidence for no effect: examples from the SIPS project. Addiction, 113(2), 240-246.

Dienes, Z., \& Mclatchie, N. (2018). Four reasons to prefer Bayesian analyses over significance testing. Psychonomic bulletin \& review, 25(1), 207-218.

Feld, G. B., \& Born, J. (2017). Sculpting memory during sleep: concurrent consolidation and forgetting. Current Opinion in Neurobiology, 44, 20-27. 
Fenn, K. M., Nusbaum, H. C., \& Margoliash, D. (2003). Consolidation during sleep of perceptual learning of spoken language. Nature, 425(6958), 614-616.

Forstmeier, W., Wagenmakers, E. J., \& Parker, T. H. (2017). Detecting and avoiding likely false-positive findings-a practical guide. Biological Reviews, 92(4), 1941-1968.

Gais, S., Lucas, B., \& Born, J. (2006). Sleep after learning aids memory recall. Learning and Memory, 13(3), 259-262.

Gorgolewski, K., Esteban, O., Schaefer, G., Wandell, B., \& Poldrack, R. (2017). OpenNeuro - a free online platform for sharing and analysis of neuroimaging data. Organization for Human Brain Mapping. Vancouver, Canada, 1677.

Hallgato, E., Győri-Dani, D., Pekár, J., Janacsek, K., \& Nemeth, D. (2013). The differential consolidation of perceptual and motor learning in skill acquisition. Cortex, 49(4), 1073-1081.

Jacoby, L. L. (1991). A process dissociation framework: Separating automatic from intentional uses of memory. Journal of Memory and Language, 30, 513-541.

Jarosz, A. F., \& Wiley, J. (2014). What are the odds? A practical guide to computing and reporting Bayes factors. The Journal of Problem Solving, 7(1), 2.

Keisler, A., Ashe, J., \& Willingham, D. T. (2007). Time of day accounts for overnight improvement in sequence learning. Learning and Memory, 14(10), 669-672.

King, B. R., Hoedlmoser, K., Hirschauer, F., Dolfen, N., \& Albouy, G. (2017). Sleeping on the motor engram: the multifaceted nature of sleep-related motor memory consolidation. Neuroscience and Biobehavioral Reviews, 80, 1-22.

Lewis, P. A., \& Durrant, S. J. (2011). Overlapping memory replay during sleep builds cognitive schemata. Trends in cognitive sciences, 15(8), 343-351.

Mantua, J. (2018). Sleep Physiology Correlations and Human Memory Consolidation: Where Do We Go From Here? Sleep, 41(2), zsx204. 
Mednick, S. C., Cai, D. J., Shuman, T., Anagnostaras, S., \& Wixted, J. T. (2011). An opportunistic theory of cellular and systems consolidation. Trends in Neurosciences, 34(10), 504-514.

Mednick, S. C., Makovski, T., Cai, D., \& Jiang, Y. V. (2009). Sleep and rest facilitate implicit memory in a visual search task. Vision Research, 49(21), 2557-2565.

Nemeth, D., Janacsek, K., Király, K., Londe, Z., Németh, K., Fazekas, K., . . Csányi, A. (2013). Probabilistic sequence learning in mild cognitive impairment. Frontiers in Human Neuroscience, 7, 318. doi:10.3389/fnhum.2013.00318

Nemeth, D., Janacsek, K., Londe, Z., Ullman, M. T., Howard, D. V., \& Howard, J. H., Jr. (2010). Sleep has no critical role in implicit motor sequence learning in young and old adults. Experimental Brain Research, 201(2), 351-358. doi:10.1007/s00221-009-2024$\mathrm{X}$

Nissen, C., Kloepfer, C., Feige, B., Piosczyk, H., Spiegelhalder, K., Voderholzer, U., \& Riemann, D. (2011). Sleep-related memory consolidation in primary insomnia. Journal of Sleep Research, 20(1 Pt 2), 129-136.

Nissen, M. J., \& Bullemer, P. (1987). Attentional requirements of learning: Evidence from performance measures. Cognitive Psychology, 19, 1-32.

Nosek, B. A., Alter, G., Banks, G. C., Borsboom, D., Bowman, S. D., Breckler, S. J., . . Christensen, G. (2015). Promoting an open research culture. Science, 348(6242), $1422-1425$.

Pan, S. C., \& Rickard, T. C. (2015). Sleep and motor learning: is there room for consolidation? Psychological Bulletin, 141(4), 812.

Payne, J. D., Kensinger, E. A., Wamsley, E. J., Spreng, R. N., Alger, S. E., Gibler, K., . . Stickgold, R. (2015). Napping and the selective consolidation of negative aspects of scenes. Emotion, 15(2), 176. 
Piosczyk, H., Holz, J., Feige, B., Spiegelhalder, K., Weber, F., Landmann, N., . . . Voderholzer, U. (2013). The effect of sleep-specific brain activity versus reduced stimulus interference on declarative memory consolidation. Journal of Sleep Research, 22(4), 406-413.

Poldrack, R. A., \& Gorgolewski, K. J. (2017). OpenfMRI: Open sharing of task fMRI data. Neuroimage, 144, 259-261.

Press, D. Z., Casement, M. D., Pascual-Leone, A., \& Robertson, E. M. (2005). The time course of off-line motor sequence learning. Cognitive Brain Research, 25(1), 375-378.

Rasch, B., \& Born, J. (2013). About sleep's role in memory. Physiological Reviews, 93(2), 681-766. doi:10.1152/physrev.00032.2012

Rickard, T. C., Cai, D. J., Rieth, C. A., Jones, J., \& Ard, M. C. (2008). Sleep does not enhance motor sequence learning. Journal of Experimental Psychology: Learning, Memory, and Cognition; Journal of Experimental Psychology: Learning, Memory, and Cognition, 34(4), 834.

Rieth, C. A., Cai, D. J., McDevitt, E. A., \& Mednick, S. C. (2010). The role of sleep and practice in implicit and explicit motor learning. Behavioural Brain Research, 214(2), 470-474.

Robertson, E. M., Pascual-Leone, A., \& Press, D. Z. (2004). Awareness modifies the skilllearning benefits of sleep. Current Biology, 14(3), 208-212. doi:10.1016/j.cub.2004.01.027

Robertson, E. M., Press, D. Z., \& Pascual-Leone, A. (2005). Off-line learning and the primary motor cortex. Journal of Neuroscience, 25(27), 6372-6378.

Rosenzweig, I., Glasser, M., Polsek, D., Leschziner, G. D., Williams, S. C., \& Morrell, M. J. (2015). Sleep apnoea and the brain: a complex relationship. The Lancet Respiratory Medicine, 3(5), 404-414. 
Rouder, J. N. (2014). Optional stopping: No problem for Bayesians. Psychonomic bulletin \& review, 21(2), 301-308.

Rouder, J. N., Speckman, P. L., Sun, D., Morey, R. D., \& Iverson, G. (2009). Bayesian t tests for accepting and rejecting the null hypothesis. Psychonomic bulletin \& review, 16(2), 225-237. doi:doi:10.3758/pbr.16.2.225

Saletin, J. M., \& Walker, M. P. (2012). Nocturnal mnemonics: sleep and hippocampal memory processing. Frontiers in Neurology, 3, 59.

Schapiro, A. C., McDevitt, E. A., Rogers, T. T., Mednick, S. C., \& Norman, K. A. (2018). Human hippocampal replay during rest prioritizes weakly learned information and predicts memory performance. Nature communications, 9(1), 3920.

Schichl, M., Ziberi, M., Lahl, O., \& Pietrowsky, R. (2011). The influence of midday naps and relaxation-hypnosis on declarative and procedural memory performance. Sleep and Hypnosis, 13(1-2), 7-14.

Schmidt, C., Collette, F., Cajochen, C., \& Peigneux, P. (2007). A time to think: circadian rhythms in human cognition. Cognitive Neuropsychology, 24(7), 755-789.

Schönauer, M., Geisler, T., \& Gais, S. (2014). Strengthening procedural memories by reactivation in sleep. Journal of Cognitive Neuroscience, 26(1), 143-153.

Schönbrodt, F. D., Wagenmakers, E.-J., Zehetleitner, M., \& Perugini, M. (2017). Sequential hypothesis testing with Bayes factors: Efficiently testing mean differences. Psychological Methods, 22(2), 322.

Scullin, M. K. (2013). Sleep, memory, and aging: the link between slow-wave sleep and episodic memory changes from younger to older adults. Psychology and Aging, 28(1), 105.

Siegel, J. M. (2001). The REM sleep-memory consolidation hypothesis. Science, 294(5544), 1058-1063. 
Sigman, M., \& Dehaene, S. (2005). Parsing a cognitive task: a characterization of the mind's bottleneck. PLoS Biology, 3(2), e37.

Simor, P., Gombos, F., Blaskovich, B., \& Bódizs, R. (2017). Long-range alpha and beta and short-range gamma EEG synchronization distinguishes phasic and tonic REM periods. Sleep, 41(3), zsx210.

Simor, P., van Der Wijk, G., Gombos, F., \& Kovács, I. (2019). The paradox of rapid eye movement sleep in the lights of oscillatory activity and cortical synchronization during phasic and tonic microstates. Neuroimage, 116066.

Simor, P., Zavecz, Z., Horvath, K., Elteto, N., Török, C., Pesthy, O., . . Nemeth, D. (2019). Deconstructing procedural memory: Different learning trajectories and consolidation of sequence and statistical learning. Frontiers in Psychology, 9, 2708.

Song, S., \& Cohen, L. G. (2014). Practice and sleep form different aspects of skill. Nature communications, 5, 3407.

Song, S., Howard, J. H., Jr., \& Howard, D. V. (2007). Sleep does not benefit probabilistic motor sequence learning. Journal of Neuroscience, 27(46), 12475-12483. doi:10.1523/jneurosci.2062-07.2007

Stickgold, R. (2013). Parsing the role of sleep in memory processing. Current Opinion in Neurobiology, 23(5), 847-853.

Stickgold, R., \& Walker, M. P. (2005). Memory consolidation and reconsolidation: what is the role of sleep? Trends in Neurosciences, 28(8), 408-415.

Stickgold, R., \& Walker, M. P. (2013). Sleep-dependent memory triage: Evolving generalization through selective processing. Nature Neuroscience, 16(2), 139-145.

Talamini, L. M., Nieuwenhuis, I. L., Takashima, A., \& Jensen, O. (2008). Sleep directly following learning benefits consolidation of spatial associative memory. Learning and Memory, 15(4), 233-237. 
Tononi, G., \& Cirelli, C. (2006). Sleep function and synaptic homeostasis. Sleep Medicine Reviews, 10(1), 49-62. doi:10.1016/j.smrv.2005.05.002

Tononi, G., \& Cirelli, C. (2014). Sleep and the price of plasticity: from synaptic and cellular homeostasis to memory consolidation and integration. Neuron, 81(1), 12-34.

Török, B., Janacsek, K., Nagy, D. G., Orbán, G., \& Nemeth, D. (2017). Measuring and filtering reactive inhibition is essential for assessing serial decision making and learning. Journal of Experimental Psychology: General, 146(4), 529.

Tucker, M., McKinley, S., \& Stickgold, R. (2011). Sleep optimizes motor skill in older adults. Journal of the American Geriatrics Society, 59(4), 603-609.

Tucker, M., Morris, C. J., Morgan, A., Yang, J., Myers, S., Pierce, J. G., . . Scheer, F. A. (2017). The relative impact of sleep and circadian drive on motor skill acquisition and memory consolidation. Sleep, 40(4), zsx036.

Vertes, R. P., \& Siegel, J. M. (2005). Time for the sleep community to take a critical look at the purported role of sleep in memory processing. Sleep, 28(10), 1228-1229.

Viczko, J., Sergeeva, V., Ray, L. B., Owen, A. M., \& Fogel, S. M. (2018). Does sleep facilitate the consolidation of allocentric or egocentric representations of implicitly learned visual-motor sequence learning? Learning and Memory, 25(2), 67-77.

Vorster, A. P., \& Born, J. (2015). Sleep and memory in mammals, birds and invertebrates. Neuroscience and Biobehavioral Reviews, 50, 103-119.

Wagenmakers, E.-J., Marsman, M., Jamil, T., Ly, A., Verhagen, J., Love, J., . . Epskamp, S. (2018). Bayesian inference for psychology. Part I: Theoretical advantages and practical ramifications. Psychonomic bulletin \& review, 25(1), 35-57.

Wagner, U., Hallschmid, M., Rasch, B., \& Born, J. (2006). Brief sleep after learning keeps emotional memories alive for years. Biological Psychiatry, 60(7), 788-790. 
Walker, M. P. (2005). A refined model of sleep and the time course of memory formation. Behavioral and Brain Sciences, 28(1), 51-104.

Walker, M. P., Brakefield, T., Hobson, J. A., \& Stickgold, R. (2003). Dissociable stages of human memory consolidation and reconsolidation. Nature, 425(6958), 616-620.

Wallace, A., \& Bucks, R. S. (2013). Memory and obstructive sleep apnea: a meta-analysis. Sleep, 36(2), 203-220.

Wamsley, E. J. (2019). Memory consolidation during waking rest. Trends in cognitive sciences, 23(3), 171-173.

Wilson, J. K., Baran, B., Pace-Schott, E. F., Ivry, R. B., \& Spencer, R. (2012). Sleep modulates word-pair learning but not motor sequence learning in healthy older adults. Neurobiology of Aging, 33(5), 991-100. 